\title{
A comparison of two methods of prognostic typing in breast cancer
}

\author{
D. R. TURNER AND C. L. BERRY \\ From the Department of Pathology, Guy's Hospital Medical School, London
}

SYNOPSIS The value of two different systems for the prognostic assessment of breast cancer has been tested by comparing a group of 74 long-term survivors with a group of 54 short-term survivors. The system devised by Bloom and Richardson (1957) gave a good general correlation with prognosis. Appearances described by Harveit (1971) showed some correlation with prognosis but were rarely seen in our cases. The degree of lympho-plasmacytic infiltrate did not show any correlation with prognosis.

Hartveit (1971) has recently described a particular morphological appearance in cases of breast carcinoma which had recurred more than 10 years after surgical treatment to the primary tumour. In such cases there were areas of tumour where the cells had clearly defined cell borders, an absence of nuclear crowding and lobulation, and a nuclear diameter of less than half the cell diameter. She found that these appearances were associated with a good prognosis and devised a grading system based on these characteristics.

Preliminary examination of a few cases of carcinoma of the breast suggested that this system was not readily applicable in clinical practice. We have therefore compared our results using Hartveit's system with those obtained using the criteria defined by Bloom and Richardson (1957). Their system depends on the degree of tubule formation, the pleomorphism of the nuclei, and the number of hyperchromatic and dividing nuclei seen. The subject of grading mammary carcinoma in general was reviewed by Bloom and Richardson (1957) who presented their findings, showing good correlation between histological grading and prognosis in cases of comparable clinical staging.

In addition, we have noted the presence or absence of a lympho-plasmacytic infiltrate in our cases. Hamlin (1968) has found that in general tumours with lymphoid infiltration have a better prognosis, and the good prognosis of medullary carcinoma is well documented (Moore and Foote, 1949; Richardson, 1956). It was considered the lymphoid infiltration should be assessed since it might be an important Received for publication 10 October 1972. factor influencing the outcome in cases of comparable staging.

\section{Materials and Methods}

Two groups of patients were selected from the files of our breast clinic, one of which comprised $\mathbf{8 0}$ patients surviving 10 years after radical mastectomy, the other 57 patients dying within five years of the same surgical procedure. Only cases which had been classified as stage I on clinical assessment before surgery and subsequently confirmed by histological assessment were included in the study in order to ensure comparability of the two groups.

Sections of paraffin-embedded material stained with haematoxylin and eosin from each case were randomized by a secretary and given a new series number. They were then classified separately by each author according to the criteria suggested by Bloom and Richardson (1957), Hartveit (1971), and also according to the presence or absence of a dense lympho-plasmacytic infiltrate within and around the tumour.

Bloom and Richardson placed the tumours in one of three grades of malignancy, namely, low (grade I), intermediate (grade II), or high (grade III). Three different aspects of the histological appearance were considered; the degree of tubule formation, the degree of nuclear pleomorphism, and the frequency of hyperchromatic nucleic and mitotic figures. Each of these factors was awarded 1, 2, or 3 points according to the degree of differentiation of the tumour. The points were added together and converted to the corresponding grade according to a 
scheme where 3,4 , or 5 points places the tumour into grade I, 6 or 7 points into grade II, and 8 or 9 points into grade III.

Hartveit's system depends upon the following cytological characteristics: clarity of cell borders, degree of nuclear crowding, degree of nuclear lobulation, and the proportion of the cell diameter occupied by the nucleus. The first three criteria were recorded as,++- , or - , and the fourth was given a numerical value of 1,2 , or 3 depending upon whether the nuclear:cytoplasmic ratio was greater than, equal to, or less than $50 \%$.

According to her system, if an area of growth in situ anywhere in the carcinoma can be classified as +--3 (or only differing from this by one grade) then the tumour is included in her type III (Figure 1) or good prognosis category. Conversely -++1 (or only differing by one grade) defined the type I (Fig. 2) or poor prognosis group. All other tumours are classified as type II.

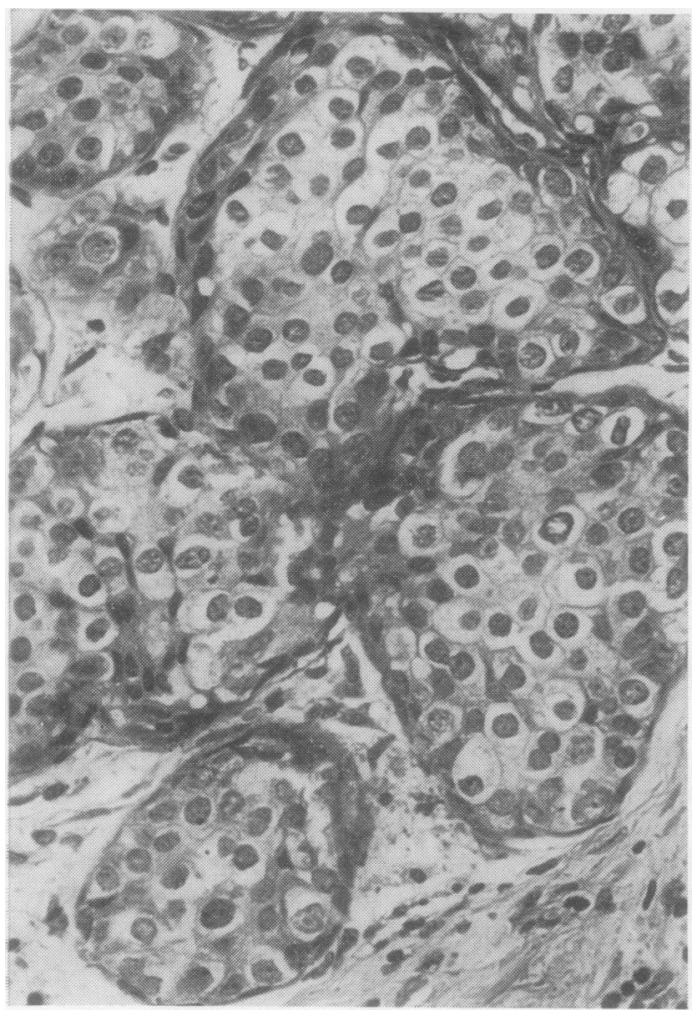

Fig. 1 Photomicrograph of Hartveit's type III good prognosis category showing well defined cell margins, a lack of nuclear crowding and nuclear lobulation, and a nuclear/cyloplasmic ratio of less than $50 \%$. $\times 750$.

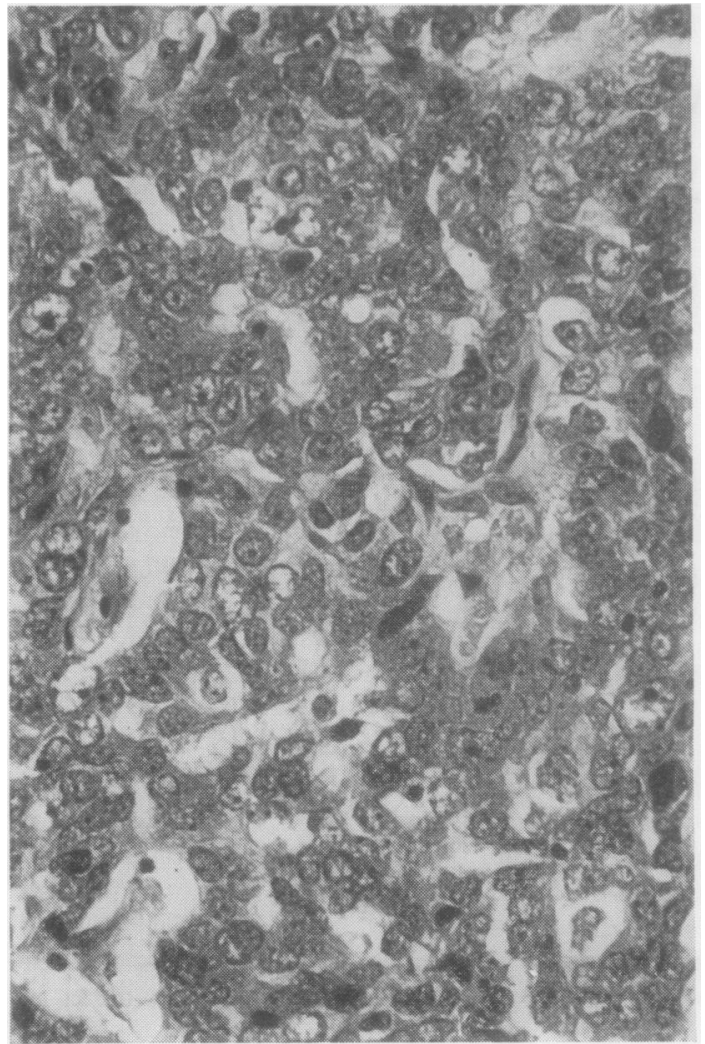

Fig. 2 Photomicrograph of Hartveit's type I poor prognosis category with poorly defined cell borders, marked nuclear crowding and lobulation, and a high nuclear to cytoplasmic ratio. $\times 750$.

\section{Results}

A preliminary survey of the tumours in our series showed two duct papillomas, one nipple adenoma, and six cases of intraduct carcinoma. These cases have not been considered in the results.

The results of classifying the remaining 74 longterm survivors and 54 short-term survivors are shown in Table I. Tumours which were classified as grade I according to Bloom's system formed a significant proportion $(39 \%)$ of the long-term survivors while only $14 \%$ with this morphology were short-term survivors. Conversely the grade III poor-prognosis category was uncommon $(16 \%)$ in long-term survivors but relatively common $(40 \%)$ in short-term survivors. The intermediate grade II tumours were divided equally between the longand short-term survivors.

Only two cases in this series fulfilled the morpho- 


\begin{tabular}{|c|c|c|c|c|c|c|c|c|c|}
\hline \multicolumn{5}{|c|}{ Bloom and Richardson (1957) } & \multicolumn{5}{|c|}{ Hartveit $(1971)$} \\
\hline Survival Time & Grade I & Grade II & Grade III & Total & Survival Time & Type III & Type II & Type I & Total \\
\hline $\begin{array}{l}>5 \mathrm{yr} \\
>10 \mathrm{yr}\end{array}$ & $\begin{array}{r}8(14 \%) \\
29(39 \%)\end{array}$ & $\begin{array}{l}25(46 \%) \\
33(45 \%)\end{array}$ & $\begin{array}{l}21(40 \%) \\
12(16 \%)\end{array}$ & $\begin{array}{l}54 \\
74\end{array}$ & $\begin{array}{l}<5 \mathrm{yr} \\
>10 \mathrm{yr}\end{array}$ & $\begin{array}{l}0(0 \%) \\
2(3 \%)\end{array}$ & $\begin{array}{r}9(17 \%) \\
26(35 \%)\end{array}$ & $\begin{array}{l}45(83 \%) \\
46(62 \%)\end{array}$ & $\begin{array}{l}54 \\
74\end{array}$ \\
\hline
\end{tabular}

Table I Gradings related to survival times in malignant disease ${ }^{1}$

${ }^{1}$ It should be noted that Hartveit type III tumours are her good prognosis category in contrast to grade I of Bloom and Richardson, good prognosis group.

logical criteria described by Hartveit for inclusion in her good-prognosis category (type III tumours). Thus in our series this appearance is a rare finding, occurring in only $3 \%$ of long-term survivors and not at all in short-term survivors. Thirty-five per cent of long-term survivors were placed in her intermediate type II category but only $17 \%$ of the short-term survivors were so classified.

Twenty per cent (11 of 54) of short-term survivors showed lympho-plasmacytic infiltration and $20 \%$ (15 of 75) of long-term survivors also showed this change.

\section{Discussion}

All systems of histological grading are based on the hypothesis that the morphological appearance of a tumour is an important factor is determining its biological behaviour. One important variable which has a marked effect on prognosis is the degree of spread of tumour when the patient is first seen. We therefore confined our study to breast tumours which had been assessed clinically as stage I before surgery. Our results show that Bloom's method of histological grading gives a good general correlation with prognosis.

Hartveit originally proposed that the area of the tumour to be examined in the assessment of prognosis should be an area of growth in situ and we adhered to this where possible. However, we were unhappy on biological grounds, for although there is a tendency for the cytological appearance of cells in breast cancer to be similar throughout the tumour, this is not always so. Hartveit's system defines a cell population in her type III tumours which is exceptionally well differentiated in terms of the conventional histological assessment of malignancy. It was found to be a rare occurrence in our series and therefore of little value for prognosis. It is interesting that some forms of lobular carcinoma in situ described by Rosen, Snyder, Foote, and
Wallace (1970) and McDivitt, Hutter, Foote, and Stewart (1967) show an appearance similar to that described by Hartveit and have a $100 \%$ cure rate when treated by radical mastectomy. In her type II category there was a considerable excess of longterm survivors indicating that there is a tendency for better differentiated tumours to be associated with longer survival. However, in her type I category there was no useful discrimination between longand short-term survivors.

The apparent lack of correlation of lymphocytic infiltration of the tumours with prognosis is at variance with the concept of immune functions having an influence on the prognosis of patients with malignant disease. However, this may be because the degree of infiltration of the primary tumour by lymphocytes is a poor method for demonstrating antitumour immunity, and we have adopted a deliberately simple yes/no classification.

In general, systems of histological grading must be simple and accurate if they are to be useful. The method of Bloom and Richardson satisfies these criteria and is apparently reproducible between different observers.

\section{References}

Bloom, H., and Richardson, W. (1957). Histological grading and prognosis in breast cancer. Brit. J. Cancer, 11, 359-377.

Hamlin, I. M. E. (1968). Possible host resistance in carcinoma of the breast: a histological study. Brit. J. Cancer, 22, 383-401.

Hartveit, F. (1971). Prognostic typing in breast cancer. Brit. med. J., 4, 253-257.

McDivitt, R. W., Hutter, R. V. P., Foote, F. W., Jr., and Stewart, F. W. (1967). In situ lobular carcinoma. J. Amer. med. Ass., 201, 82-86.

Moore, O. S., and Foote, F. W., Jr. (1949). The relatively favorable prognosis of medullary carcinoma of the breast. Cancer (Philad.), 2, 635-642.

Rosen, P., Snyder, R. E., Foote, F. W., and Wallace, T. (1970). Detection of occult carcinoma in the apparently benign breast biopsy through specimen radiography. Cancer (Philad.), 26, 944-952.

Richardson, W. W. (1956). Medullary carcinoma of the breast: a distinctive tumour type with a relatively good prognosis following radical mastectomy. Brit. J.Cancer, 10,415-423. 